\title{
Constant Envelope FrFT- OFDM with Physical Layer Security
}

\author{
Dida Mussa $\mathrm{A}^{+}$, Hao Huan, Wang Teng, and Ran Tao \\ Department of Electronic Engineering, Beijing Institute of Technology, Beijing, China
}

\begin{abstract}
Physical layer security uses channel characteristics to increases security of the communicating entities. In this paper, channel signature were used to improve the security of wireless communications uses constant envelope with fractional Fourier transform OFDM (CE-FrFT-OFDM) as its multiplexing technique instead of traditional OFDM. The chosen technique is power efficient and poses the same implementation complexity and performance as traditional OFDM. The physical layer security proposed is easily deployed in CE-FrFT-OFDM than in OFDM and maintains its performance advantages. The proposed system secure a communication system by dynamic arrangement of subcarriers (DAS) based on the average channel gain of the subcarriers. The random variation of the wireless channel and its reciprocity between the transmitter and the legal receiver ensures that the communicating entities shares the same channel impulse response (CIR). The DAS also reduces the signal correlation between the communication pairs $40 \%$ while that of transmittereavesdropper is reduced by $95 \%$ and it increases the computation cost of the eavesdropper in obtaining the transmitted information which leaves guessing of the channel gain the only viable choice of obtaining the transmitted information. By increasing the accepted lever of a channel gain we can increase the secrete capacity of the communication system while maintaining CE-FrFT-OFDM advantages.
\end{abstract}

Keywords: Physical Layer security, dynamic arrangement of subcarriers (DAS), Fractional Fourier Transform (FrFT), FrFT-OFDM, and Constant Envelope.

\section{Introduction}

Securing a wireless communication is a challenging task mainly due to its broadcast nature and standardisation of the communication systems. It's broadcast nature allows any device within the range of communication to easily eavesdrop the communication while the equipment and technological standardisations helps the eavesdropper in obtaining signal parameters and decode the transmitted information [1],[2]. To secure a communication system, upper layer encryption schemes were used, leading to reduced system throughput for additional encryption information required. In the contrary, Physical layer encryption exploits physical characteristics of the wireless channel to secures a communication system without trading-off system's throughput and can easily integrated with higher level security measures [3]-[5].

Different approaches have been proposed to address the security of wireless communication at the physical layer level including sorting of subcarriers, relay antennas or noise enhancing encryption [6]-[8]. In recent researches wireless link signature is used to secure the communication since it can easily categorizes different receiver based on their physical locations [6].

Link signature is the unique transmitter-legal receiver wireless channel characteristics; it can easily authenticate communicating entities in a given time frame. Its random variation pose a challenge for an eavesdropper to accurately obtain it within a communication period. In this paper, the mean channel gain of the predefined CE-FrFT-OFDM subcarriers is used as a link signature to secure a wireless communication system.

\footnotetext{
+ Corresponding author. Tel.: + 8618811789643.

E-mail address: mussa.ally@nm-aist.ac.tz..
} 
The one sides (imaginary) of CE-FrFT-OFDM subcarriers is used to calculate the channel gain. The gain is then used to dynamically arrange the subcarriers of the other side (real). The extension of CE-FrFT-OFDM subcarriers to double its subcarriers, its fast implementation using FrFT, OdB PAPR, and high SER and low inter-carrier interference (ICI) in time-frequency varying environment are factors which contributed to its use in this study[9], [10].

The DAS employed is a simplified version of the one provided in [11] and [12]. Although the proposed scheme can also works for conventional OFDM and other multicarrier systems but the signal extension in CEFrFT-OFDM provides extra advantage for securing the transmitted signal as only the extended real subcarriers of the transmitted information are scrambled and the other side is used to obtain the channel impulse response (CIR) for the receiver to re-arrange the transmitted signal.

The rest of the paper is organised as follows; Section II gives the details of the system description developed and how security improvement is achieved. Section III covers analysis of the Physical layer security improvement offered by the system. Section IV gives analytical security improvement offered by the system. Section V provides the simulation results and its analysis, and Section VI concludes this paper

\section{Proposed Physical layer security}

The CE-FrFT-OFDM system is detailed explained by our earlier work in [9], [10]. In securing the transmitted information using physical layer characteristics as shown in Fig. 1 the following assumptions were made:

- Full duplex communication between transmitter and receiver,

- There is channel reciprocity between transmitter and legal receiver,

- Availability of passive eavesdropper who silently listen to communication, and

The channel estimation is performed in frequency domain and real sided pilot subcarriers of CE-FrFTOFDM are equalized with the imaginary side to get accurate channel signature for DSA and re-ranging. The DAS is performed by;

$$
m_{i}^{a}=\bmod _{N}\left(B \cdot \bar{h} \cdot m_{i}+C\right)
$$

where $m_{i}^{a}$ is arranged subcarrier, $\bmod _{N}$ is module $N$ operation, $N$ is number of subcarriers, $\bar{h}$ is average channel attenuation given by, $m_{i}$ is unscrambled subcarrier, $B$ and $C$ are constants.

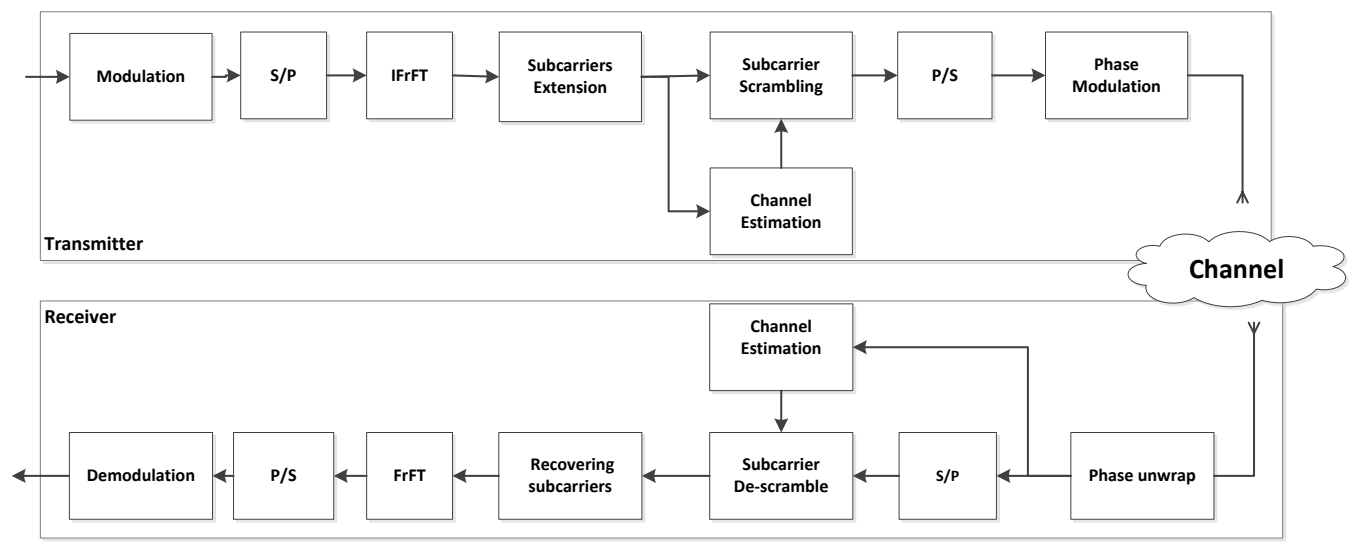

Fig. 1: Physical layer security using CE-FrFT-OFDM

Then the subcarriers are parallel to serially converted and wrapped together to form a CE-FrFT-OFDM ready for transmission. The details of obtaining $h$ is obtained in [16] and the proposed DSA algorithm is given by;.

The DSA algorithm;

Constants: Number of Subcarriers, B and C.

Variable: Channel attenuation (from channel estimation)

- Start counter,

- Receive the channel attenuation 
- Format the channel attenuation based on the required format as given by (2)

- Dynamically arrange the subcarriers based on (1)

- Decrement counter

- Check counter state $\{$ if counter $=0$ go to 1 , else go to 3$\}$

$$
\bar{h}_{m}=\frac{1}{g}\left\lfloor g \cdot \bar{h}_{m}\right\rfloor
$$

where $0<\bar{h}_{m} \leq 1, g \geq 10$ and it is constant used to approximate correct $\bar{h}_{m}$, and $\lfloor\cdot\rfloor$ calculates a minimum integer.

Table 1: Example of Subcarrier mapping

\begin{tabular}{|c|c|c|c|c|c|c|c|c|c|c|c|c|c|c|c|c|c|}
\hline \multirow{5}{*}{ 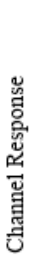 } & & \multicolumn{16}{|c|}{ Subcarriers Mappings } \\
\hline & & 0 & 1 & 2 & 3 & 4 & 5 & 6 & 7 & 8 & 9 & 10 & 11 & 12 & 13 & 14 & 15 \\
\hline & 0.7 & 3 & 10 & 1 & 8 & 15 & 6 & 13 & 4 & 11 & 2 & 9 & 0 & 7 & 14 & 5 & 12 \\
\hline & 0.9 & 3 & 14 & 9 & 4 & 15 & 10 & 5 & 0 & 11 & 6 & 1 & 12 & 7 & 2 & 13 & 8 \\
\hline & 1.1 & 3 & 0 & 13 & 10 & 7 & 4 & 1 & 14 & 11 & 8 & 5 & 2 & 15 & 12 & 9 & 6 \\
\hline
\end{tabular}

\section{Performance analysis}

For a transmitted signal $x(t)$, a legal receiver will receive $y_{m}(t)$ which is a function of transmitter-legal receiver channel attenuation, $h_{m}$ referred as main channel and the corresponding AWGN noise $w_{m}$. The eavesdropper can also detect the signal as $y_{e}(t)$ and it is the version of the transmitted signal and affected by channel attenuation between transmitter and eavesdropper, $h_{e}$ referred as eavesdropper channel and its corresponding AWGN $w_{e}$ as represented by;

$$
\begin{aligned}
& y_{m}(t)=h_{m} \otimes x(t)+w_{m} \\
& y_{e}(t)=h_{e} \otimes x(t)+w_{e}
\end{aligned}
$$

where $\otimes$ is convolution operation.

For multipath channel, the channel response depends on type of environment and distance between the transmitter and receiver. For a stationary or slowly moving receiver or transmitter $h_{m} \alpha \frac{1}{d_{m}{ }^{n}}$ and $h_{e} \alpha \frac{1}{d_{e}{ }^{n}}$ where $d_{m}$ is distance between transmitter and legal receiver, and $d_{e}$ is the transmitter-eavesdropper distance, and $n$ is path loss exponent.

The average channel gain calculated using least square (LS) or minimum mean square error (MMSE) and approximate it using (2) for DSA. In calculating channel gain based on (2), the average channel gain from the first received signal is obtained from each pilot subcarriers. The maximum allowed gain separation $\varsigma$ depends on the allowed number of channel gain $M$

$$
\varsigma=\bar{h}_{m}^{2}-\bar{h}_{m}^{1}=\bar{h}_{m}^{3}-\bar{h}_{m}^{2}=\frac{\bar{h}_{m}^{\max }-\bar{h}_{m}^{\min }}{M}
$$

where $M$ is a total number of acceptable channel gains, and $\varsigma$ is the maximum allowed difference between the coefficients used for approximation and reducing signal detection errors. The receiver will be able to rearrange the subcarriers if the calculation error is less than $\varsigma$. In our experiment $M=20, F\left(\bar{h}_{m}^{\text {max }}\right)=1, F\left(\bar{h}_{m}^{\text {min }}\right)=0$, and $\varsigma=0.05$

Decreasing $\varsigma$ increases eavesdropper computations and the guessing time by $M$ times off legal receiver, so the legal receiver will calculates new $\bar{h}$ at least every $\frac{M}{2}$ of the symbol time which is close to coherence time, for a secure communication it is calculated every $\frac{M}{4}$ of a CE-FrFT-OFDM block time. 


\section{Security Analysis}

The security of the proposed system is evaluated using SER of the eavesdropper, that of legal receiver, and the secrecy capacity of the system based on the channel correlation between the legal receiver and eavesdropper.

\subsection{SER of Eavesdropper}

As discussed in Section 2, the eavesdropper can only perform random guess of the subcarrier arrangements. It easy for an eavesdropper to try guessing the channel gain used to arrange the subcarriers than the actual subcarrier arrangements. So the probability of correct guessing depends on acceptable range channel gain $M$, and time taken for the legal receiver to constantly change the arrangement order. In a given time slot $t$, the probability of an eavesdropper to correctly guess the channel signature $P_{e}$ is given by;

$$
P_{e}=\frac{1}{2 M}
$$

The lack of extra side information increases security of the transmitted information. This increases eavesdropper computations in a given time frame before new arrangement is performed. For an eavesdropper with memory, large $M$ will be preferred but the cumulative error in detecting the received signal will be increasing for each error made. If $\gamma$ is a SER of a CE-FrFT-OFDM system, then SER of an eavesdropper can be given by (6)

$$
\gamma_{e}=1-P_{e}(1-\gamma)
$$

\subsection{Reliability of the proposed system}

In detecting the received signal, channel estimation errors will lead to in accuracy of subcarrier rearrangement. The system reliability depends on the estimation errors and the maximum number of channel gain index used in the system. The estimation error $e$ is given by;

$e=h_{m}-h_{t}$
where $($.$) means estimated value of legal receiver/transmitter channel gain and e \leq \varsigma$

$$
\begin{aligned}
& e=h_{m}-h_{t} \\
& \text { eceiver/transmitter channel gain and } e \leq \varsigma
\end{aligned}
$$

The estimated $h_{m}$ that is observed by the legitimate receiver can be considered as a noisy version of $h_{t}$ that has been previously obtained by a transmitter and used to determine the interleaving pattern.

$$
\begin{gathered}
h_{m}=\bar{h}_{m}+\Delta h_{m} \\
h_{t}=\bar{h}_{m}+\Delta h_{t}
\end{gathered}
$$

The reliability of the system depends on the probability of transmitter and legal receiver to correctly estimate the required channel gain and explained in details in [6]. Our research calculated the approximation error obtained by receiver and legal receiver and transmitter based on (2) and (8) and MATLAB simulations were used for its calculation.

The SER of the legal receiver is given by (6), the channel gain error in (7) reflects the reliability of the system. By reducing it we increase system reliability and computational cost while reducing system security and a balance between computational cost, security and system reliability is needed while doing so. The legal receiver SER is given by;

$$
\gamma_{m}=1-P_{L}(1-\gamma)
$$

\subsection{Secrecy capacity}

The secrecy capacity is the difference between the capacities of main channel and eavesdropper channel as given by (10)

$$
C_{s}= \begin{cases}\log _{2}\left(1+\gamma_{m}\right)-\log _{2}\left(1+\gamma_{e}\right), & \text { if } \gamma_{m}>\gamma_{e} \\ 0 & \text {, otherwise }\end{cases}
$$
where $P$ is the source transmit power, and $\frac{|h|^{2}}{N_{o}}$ is signal to noise ration of the channel, If $P \gg N_{o}$, then the
secret capacity becomes,

$$
C_{s} \leq \log _{2}\left(\frac{P_{L}\left|h_{m}\right|^{2}}{P_{e}\left|h_{e}\right|^{2}}\right)
$$


As shown in [15], the secrecy rate depends on the correlation between the channel coefficients, By using channel gain to dynamically arrange the subcarriers we will reduce the correlation of the subcarriers for legal receiver and is reduced further for an eavesdropper as shown in section 5 and thus increase the secrecy capacity of (11).

\section{Simulation Results}

In simulating the proposed system $128 \mathrm{FrFT} / \mathrm{IFrFT}$ size were used with 1000 symbols at 8 PSK and the Rayleigh channel with $100 \mathrm{~Hz}$ Doppler frequency.

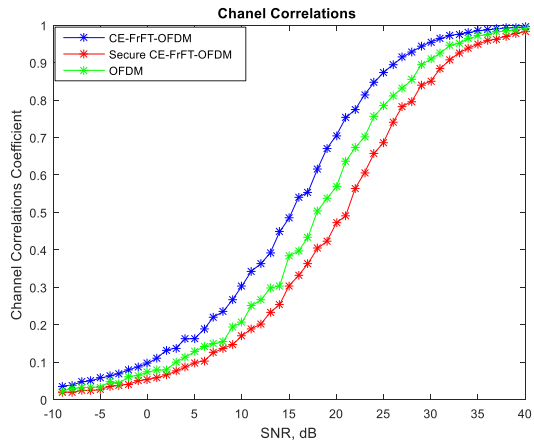

a

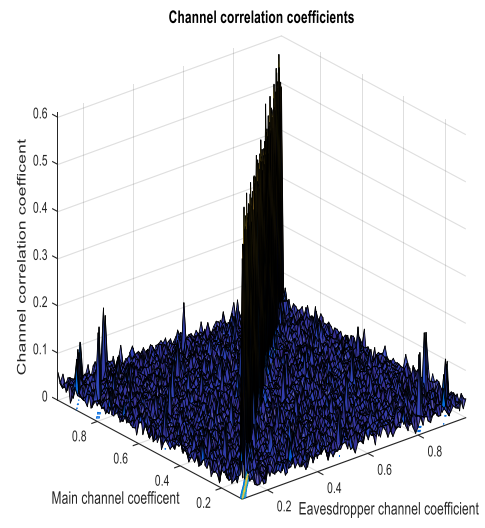

b

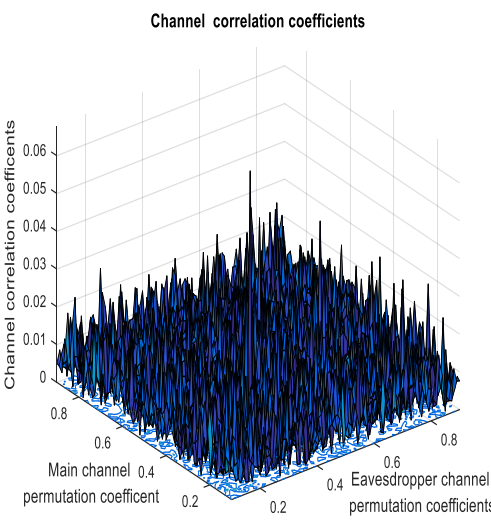

C

Fig 2: (a) Comparison of Channel correlation coefficients comparison, (b) Signal correlation coefficients for legal receiver-eavesdropper with channel information (c) Signal correlation coefficients for legal receiver-eavesdropper without channel information

Fig 2 (a) compares the channel correlation coefficients against Signal to Noise Ratio (SNR) for the secure CE-FrFT-OFDM, unsecure CE-FrFT-OFDM and traditional OFDM. The low channel correlation coefficients proves the system is secure than OFDM. Fig 2(b) and 2(c) compares the channel correlation coefficient between transmitter and legal receiver and eavesdropper when sharing the same channel (colocated) and when not sharing the channel. The correlation coefficients shows high correlation when sharing the channel Fig 2(b) and low correlation without sharing the channel Fig 2(c), this proves the eavesdropper can only correctly obtain the transmitted information when co-located with the legal receiver.

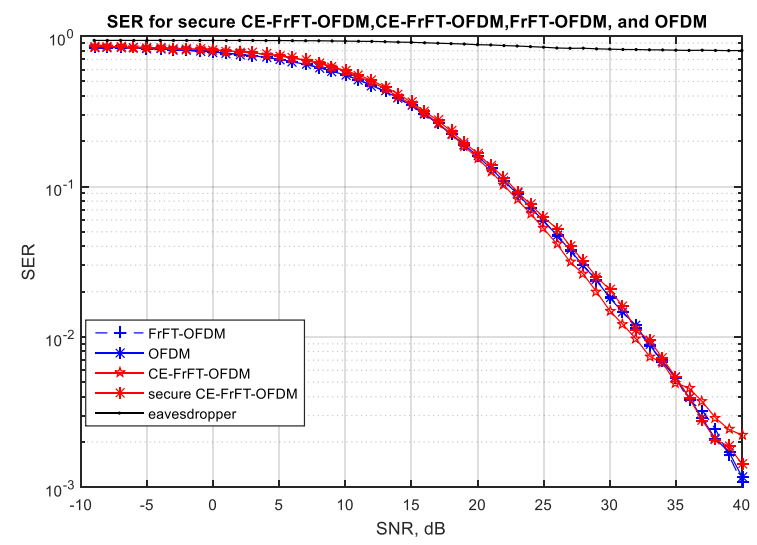

a

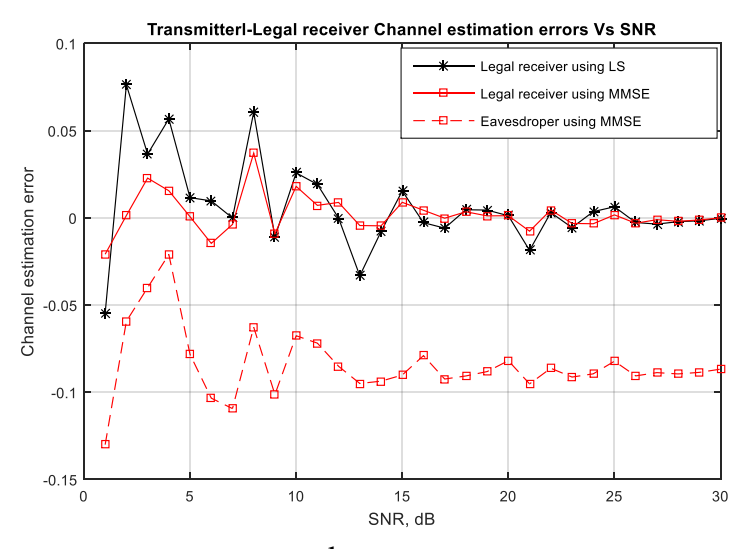

b

Fig 3: Performance of a secure CE-FrFT-OFDM (a) SER between legal receiver and eavesdropper (b) channel estimation errors

Fig 3 (a) compares the SER of the proposed system with that of an OFDM system and unsecure CEFrFT-OFDM system. The comparison shows the proposed system obtains the same performance for legal receiver and high SER for an eavesdropper. This means the security improvement offered by the proposed system does not affect system throughput and only disturb eavesdropper performance. 
Fig 3(b) shows the channel estimation errors obtained. The errors obtained are less than the maximum allowed 0.05 when using minimum mean square error (MMSE) estimator hence high reliable system. But when using least square (LS) channel estimator the system reliability is reduced at low SNR but reliability increase at high SNR. For eavesdropper, the channel estimation error is always bigger than the maximum allowed limit even in higher SNR.

\section{Conclusion}

The proposed system increases the security of wireless communication by dynamically arranging its CEFrFT-OFDM subcarriers based on channel signatures. The arrangement ensures the low channel correlation compared to OFDM. The passive eavesdropper cannot detect the transmitted signal unless it shares the receiver signature link. The proper guessing can only be obtained when the eavesdropper coexist at the same location as legal receiver otherwise cannot obtain the transmitted signal. The SER performance of the system shows the system will have the same performance as OFDM and unsecure CE-FrFT-OFDM system.

\section{References}

[1] K. Havish, A. A. Hassan, and S. Chennakeshu, "Secure Information Transmission for Mobile Radio.” 2000.

[2] Y. Aylin and U. Sennur, "Wireless Physical-Layer Security: Lessons Learned From Information Theory," Proc. IEEE, 2015.

[3] A. Mukherjee, F. S. Ali A., H. Jing, and S. A. Lee, "Principles of Physical Layer Security in Multiuser Wireless Networks: A Survey.” IEEE Communication Surveys \& Tutorials, 2014.

[4] Yulong Zou, Jia Zhu, Xianbin Wang, and Victor C.M. Leung, "Improving Physical-Layer Security in Wireless Communications Using Diversity Techniques.” IEEE Network, 2015.

[5] Xiaoming Chen and Rui Yin, "Performance Analysis for Physical Layer Security in Multi-Antenna Downlink Networks with Limited CSI Feedback.” IEEE wireless Communication Letters, 2013.

[6] H. Li, X. Wang, and J.-Y. Chouinard, "Eavesdropping-Resilient OFDM System Using Sorted Subcarrier Interleaving.” IEEE Transactions on Wireless Communications, 2015.

[7] Xiaoming Chen, Caijun Zhong, Chau Yuen, and Hsiao-Hwa Chen, "Multi-Antenna Relay Aided Wireless Physical Layer Security.” IEEE Communications Magazine, 2015.

[8] Truc Thanh Tran and Hyung Yun Kong, "CSI-Secured Orthogonal Jamming Method for Wireless Physical Layer Security.” IEEE Communication Letters, 2014.

[9] M. A. Dida, H. Hao, A. M. R., and R. Tao, "Constant Envelope Chirped OFDM Power Efficiency." 4th International Conference on Wireless and Optical Communications, 2016.

[10] M. A. Dida, H. Hao, X. Wang, and R. Tao, "Constant Envelope Chirped OFDM for Powerefficient Radar Communication.” IEEE Information Technology,Networking,Electronic and Automation Control Conference, 2016.

[11] R. Nordin, S. Armour, and J. P. McGeehan, "Dynamic Subcarrier Allocation with ESINR Metric in Correlated SM-OFDMA,” 2010 IEEE Wirel. Adv. Conf., 2010.

[12] Hao Li, Xianbin Wang, and Weikun Hou, "Secure Transmission in OFDM Systems by Using Time Domain Scrambling," 2013 IEEE 77th Veh. Technol. Conf., 2013.

[13] Junxing Zhang, Mohammad H. Firooz, Neal Patwari, and Sneha K. Kasera, “Advancing Wireless Link Signatures for Location Distinction.” international conference on Mobile computing and networking, 2008.

[14] Neal Patwari and Sneha K. Kasera, "Temporal Link Signature Measurements for Location Distinction,” IEEE Trans. Mob. Comput., vol. 10, no. 3, pp. 449-462, 2011.

[15] Hyoungsuk Jeon, Namshik Kim, Jinho Choi, Hyuckjae lee, and Jeongseok Ha, "Bounds on Secrecy Capacity Over Correlated Ergodic Fading Channels at High SNR,” IEEE Trans. Inf. Theory, vol. 57, no. 4, pp. 1975-1983, 2011. 